\title{
Cationization of Alpha-Cellulose to Develop New Sustainable Products
}

\author{
Ana Moral, ${ }^{1}$ Roberto Aguado, ${ }^{1}$ Menta Ballesteros, ${ }^{1}$ and Antonio Tijero ${ }^{2}$ \\ ${ }^{1}$ ECOWAL Group, Molecular Biology and Biochemical Engineering Department, Experimental Sciences Faculty, \\ Pablo de Olavide University, Carretera de Utrera km 1, 41013 Seville, Spain \\ ${ }^{2}$ Celulosa y Papel Group, Chemical Engineering Department, Faculty of Chemistry, Complutense University of Madrid, \\ Avenida Complutense s/n, 28040 Madrid, Spain
}

Correspondence should be addressed to Ana Moral; amoram@upo.es

Received 18 March 2015; Revised 26 May 2015; Accepted 27 May 2015

Academic Editor: Yulin Deng

Copyright (C) 2015 Ana Moral et al. This is an open access article distributed under the Creative Commons Attribution License, which permits unrestricted use, distribution, and reproduction in any medium, provided the original work is properly cited.

\begin{abstract}
Papermaking has been using high quantities of retention agents, mainly cationic substances and organic compounds such as polyamines. The addition of these agents is related to economic and environmental issues, increasing contamination of the effluents. The aim of this work is to develop a cationic polymer for papermaking purposes based on the utilization of alpha-cellulose. The cationization of mercerized alpha-cellulose with 3-chloro-2-hydroxypropyltrimethylammonium chloride (CHPTAC) is governed by a pseudo-second-order reaction. The initial amorphous fraction of cellulose is reacted with CHPTAC until the equilibrium value of nitrogen substitution is reached. Nitrogen is incorporated as a quaternary ammonium group in the polymer. Also, the kinetic constant increased with decreasing crystallinity index, showing the importance of the previous alkalization stage. The use of modified natural polysaccharides is a sustainable alternative to synthetic, nonbiodegradable polyelectrolytes and thus is desirable with a view to developing new products and new processes.
\end{abstract}

\section{Introduction}

Paper manufacturing processes require the addition of retention agents for (i) compacting the pulp, formed by anioniccharged biopolymers, and (ii) avoiding industry wastewater effluents containing high amounts of microfibers that are released from the original pulp $[1,2]$. Traditionally, papermaking has used high quantities of retention agents, mainly cationic substances such as inorganic aluminium compounds (aluminium sulphate and poly(aluminium chloride)), and/or certain organic compounds, mainly polyamines [3]. The addition of these agents is related to economic and environmental issues, as they induce the apparition of adherent flocs [4] increasing contamination of the effluents. This has awakened the scientific community's interest to explore new alternatives. In the last decades, semisynthetic compounds manufactured from natural polymers (e.g., highly cationic starch) have also been employed in papermaking industries [5-10]. Modification of natural polymers in order to achieve new materials with specific properties can be carried out by cationization. In fact, in many industries, cationic polysaccharides are replacing the traditional consumption of cationic polyacrylamides for their use as colloid flocculants, due to their lower toxicity and less legal restrictions on their consumption [11].

The cationic modification of cellulose commonly goes by the etherification with a 2-hydroxy-3-(trimethylammonium)propyl group, which can be obtained by the reaction of the biopolymer and 2,3-epoxypropyltrimethylammonium chloride (EPTAC). EPTAC, however, is an unstable and toxic reagent that cannot be used in industrial applications [11]. An alternative is the use of 3-chloro-2-hydroxypropyltrimethylammonium chloride (CHPTAC). CHPTAC has been successfully used for the cationization of cellulose [12-15], textile fibres [16-20], and other polysaccharides such as agarose or backbone of tamarind kernel polysaccharide (TKP), among others $[21,22]$. The flocculation efficiencies achieved for the resulting products are similar to those obtained with classical commercial polyacrylamides [21].

A three-step mechanism was proposed for the reaction of CHPTAC and cellulose. In the first, fast reaction, the chlorohydrin CHPTAC is converted to the epoxide EPTAC. 
Then, a hydroxyl group of the cellulose is converted into an alkoxide by reacting with a base (e.g., $\mathrm{NaOH}$ ). Finally, the alkoxide from cellulose reacts with EPTAC, resulting in cationized cellulose. A secondary reaction could occur between EPTAC and water, which must be avoided as the formed diol is unable to react with cellulose, increasing the cost of the cationic modification [11, 12, 23]. During cationization, the degree of substitution is highly influenced by the quantity of base added. This addition is necessary not only to generate EPTAC from CHPTAC, but also to weaken the hydrogen bonds between molecules, making cellulose more accessible [17]. Therefore, it can be used as a pretreatment, taking into account the fact that an excess of base favours polysaccharide hydrolysis and epoxide degradation towards the aforementioned diol.

Cellulose is a linear homopolymer composed of aD-glucopyranose units linked by $\beta$-1,4-glycosidic bonds $\left(\mathrm{C}_{6} n \mathrm{H}_{10} n+2 \mathrm{O}_{5} n+1 \quad n=\right.$ degree of polymerization of glucose)). Native cellulose is essentially cellulose I, which has a structure made of parallel chains [24]. When subjected to strong alkaline media during the alkalization-cationization process, cellulose becomes swollen and, upon washing, shrinks back to yield a new allomorph, cellulose II $[25,26]$, within the crystalline domains [27-29]. X-ray diffraction gives the most direct results for the characterization of the crystalline structure of cellulose [30-32]; however, its interpretation is still under discussion.

The objective of this work is to develop a cationic polymer for papermaking purposes based on the utilization of alpha-cellulose. X-ray measurements were used to study the crystalline structure and the kinetic equations governing the process are discussed to find out the optimal concentrations of reagents. The use of modified natural polysaccharides is currently regarded as a sustainable alternative to synthetic polymers and hence as specially desirable with a view to developing new improved products.

\section{Experimental}

2.1. Alkalization. The raw material used was commercial $\alpha$ cellulose (Aldrich C8002). Reactions took place in a $2 \mathrm{~L}$ threemouthed spherical glass reactor with a refluxing condenser, a magnetic stirrer, heating controlled by an electronic PID device, and a Pt-100 probe to measure temperature, which was kept at $25^{\circ} \mathrm{C}$. $\mathrm{NaOH}$ pellets were purchased from Aldrich. In each experiment, $25 \mathrm{~g}$ of cellulose (on the basis of dry pulp weight) was mixed with $500 \mathrm{~mL}$ of an aqueous $\mathrm{NaOH}$ solution $(10-30 \% \mathrm{w} / \mathrm{w})$. Nine alkalization experiments were carried out for different reaction times and $\mathrm{NaOH}$ concentrations (Table 1). Afterwards, fibres were separated by passage through a Whatman Glass Microfiber Binder Free Grade GF/D filter $(2.7 \mu \mathrm{m})$ and washed with demineralized water. The alkalized cellulose thus obtained was dried in a vacuum stove at $45^{\circ} \mathrm{C}$.

2.2. Cationization. The cationizing agent was an aqueous solution of 3-chloro-2-hydroxypropyltrimethylammonium chloride (CHPTAC) at $60 \%(\mathrm{w} / \mathrm{w})$ from Aldrich. The process
TABLE 1: Mercerization conditions: codification.

\begin{tabular}{lccc}
\hline Soda concentration (\%) & \multicolumn{3}{c}{ Reaction time (min) } \\
& 30 & 60 & 90 \\
\hline 10 & A1T1 & A1T2 & A1T3 \\
20 & A2T1 & A2T2 & A2T3 \\
30 & A3T1 & A3T2 & A3T3 \\
\hline
\end{tabular}

was carried out in two steps: first, formation of epoxypropyltrimethylammonium chloride (EPTAC) by reaction with hydroxide ions, and second, nucleophilic substitution of the hydroxyl group bonded to $\mathrm{C}_{6}$ in the anhydroglucose unit (AGU).

An amount of $20 \mathrm{~g}$ of alkalized cellulose (on the basis of dry pulp) was mixed with the reagent in a CHPTAC/AGU mole ratio of 4 , assuming that the material is wholly constituted by anhydroglucose units. A volume of $395 \mathrm{~mL}$ of an aqueous $\mathrm{NaOH}$ solution at $5 \%(\mathrm{w} / \mathrm{w})$ was added. Then, the mixture was completed with enough isopropyl alcohol to achieve a final volume of $1 \mathrm{~L}$. The reactor was the one used in the previous alkalization step and the temperature was held at $70^{\circ} \mathrm{C}$ through the process. The reaction timer started when the setpoint temperature was reached.

Samples from the reaction mixture were withdrawn every 30 minutes by using a $50 \mathrm{~mL}$ wide mouth pipette. Immediately, they were diluted ten times with cold water to stop the reaction, passed through a Whatman Glass Microfiber Binder Free Grade GF/D filter $(2.7 \mu \mathrm{m})$, washed with demineralized water, and dried in a vacuum stove at $45^{\circ} \mathrm{C}$. Dried samples were kept sealed at room temperature.

2.3. Characterization of Samples. Cellulose and alkalized cellulose samples were characterized by using a PANalytical powder X-ray diffractometer in combination with the X'Pert software. Adopting the two-phase theory of structure and the amorphous halo correction, the crystallinity index $(C r I)$ was obtained from the X-ray diffraction curves [33].

Carbon and nitrogen contents were determined by combustion on a LECO CNS-2000I elemental macroanalyser. Knowing the amounts of nitrogen incorporated as quaternary ammonium into cellulose by effect of the cationization treatment, the degree of substitution was determined. The carbon content was used to apply a correction to systematic errors. The results could be processed to evaluate the kinetics models $[32,34,35]$.

\section{Results and Discussion}

3.1. X-Ray Diffraction Spectroscopy. Figure 1 shows the XRD patterns for the starting cellulose and alkalized cellulose at the preset $2 \theta^{\circ}$ values used. We chose to represent the patterns in a staged manner in order to more easily envisage changes in the initial fibres by effect of the alkaline treatment. The structural changes undergone by cellulose in the treatments preceding cationization were used to examine their relation to the cationization results. The changes caused by a $\mathrm{NaOH}$ concentration of $10 \%$ were minimal, even at the longest 


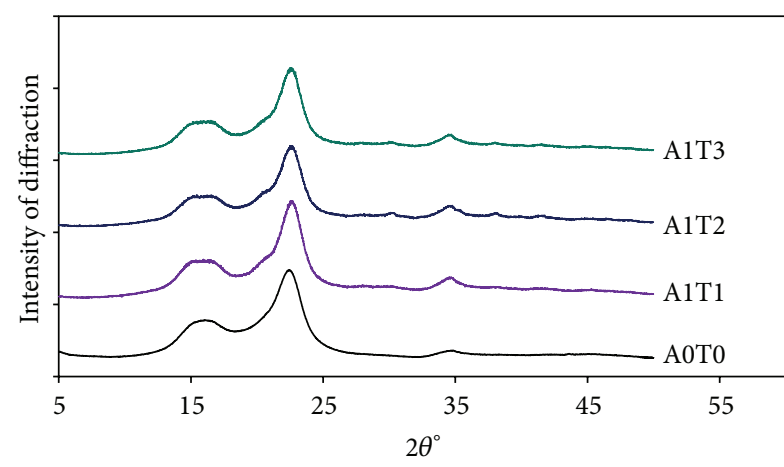

(a)

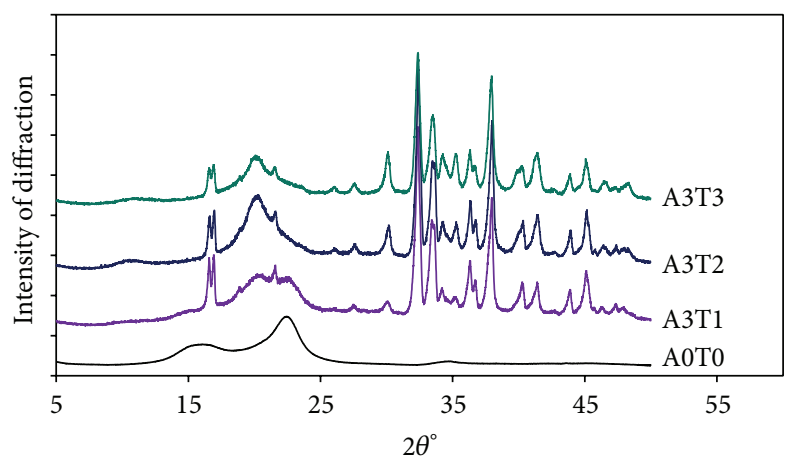

(c)

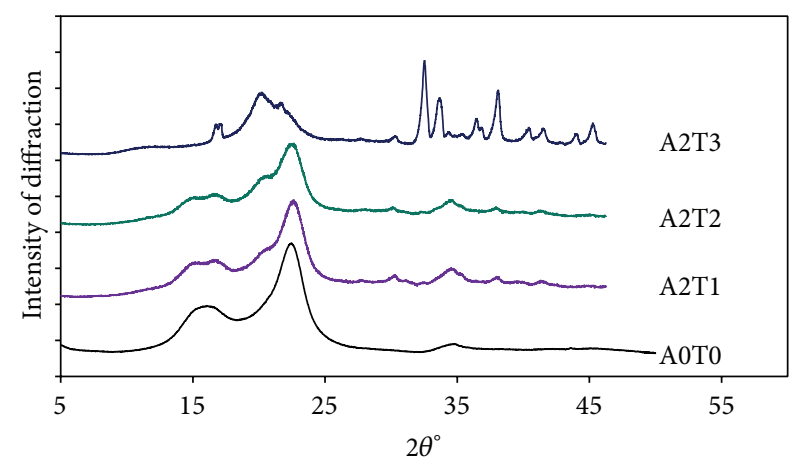

(b)

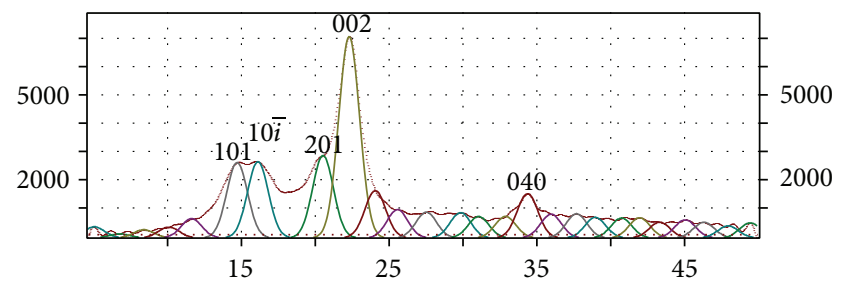

(d)

Figure 1: Diffractograms of untreated cellulose and of samples alkalized with different $\mathrm{NaOH}$ concentrations and times. (a) NaOH $10 \%$. (b) $\mathrm{NaOH} 20 \%$. (c) $\mathrm{NaOH} 30 \%$. (d) AlT1 after deconvolution.

operation times used; however, they increased as the alkali concentration was raised.

Although the enzymatic and nonenzymatic reaction are additionally influenced by other factors, we used the crystallinity index $(\mathrm{CrI})$ and the fractions of cellulose I and cellulose II in the starting and alkalized cellulose to compare and interpret the results of the cationization process.

Park et al. [36] previously examined the structure of cellulose treated with sodium hydroxide and carbon dioxide by X-ray diffraction and FTIR spectroscopy. The crystallinity index was calculated from the height ratio of the intensity at $2 \theta=22.5^{\circ}$ for cellulose $\mathrm{I}$, or the 101 reflection at $2 \theta=$ $19.8^{\circ}$ for cellulose II (crystalline height, $h c r$ ), to the height of the amorphous reflection corresponding to $2 \theta=18.8^{\circ}$ for cellulose I or $2 \theta=16.1^{\circ}$ for cellulose II (amorphous height, ham), respectively [6]. While the peak height method is widely used to measure the crystallinity index, due to its simplicity, it produces an overestimation of this index and neglects the influence of peak width [37]. We measured the proportion of cellulose I (CI) and that of cellulose II (CII) by using the peak height (see (1)), but the crystallinity index was determined following the XRD deconvolution method, and thus using the peak area:

$$
\mathrm{CI}=1-\frac{h_{\mathrm{am}}}{h_{\mathrm{cr}}}
$$

$$
\begin{gathered}
\frac{h_{\mathrm{am}}}{h_{\mathrm{cr}}}=\frac{I_{18.8 \min }}{I_{19.8 \max }+I_{22.5 \max }-I_{18.8 \min }} \\
\mathrm{CII}=\frac{I_{12.1}}{I_{12.1}+0.5\left(I_{14.6}+I_{16.1}\right)} .
\end{gathered}
$$

Gaussian deconvolution was performed with Systat's PeakFit software. The crystallinity index was calculated by dividing the sum of the areas of the five crystalline peaks $(101,10 \bar{i}, 021$, 002, and 040) by the total area [37]. Figure 1(d) shows the crystalline peaks of sample A1T1 after deconvolution, as an example.

As is shown in Figure 2, structural changes in cellulose by effect of the treatments preceding cationization were notorious under the experimental conditions used. The conversion into cationized cellulose must be governed largely by that of cellulose I into cellulose II and the latter into hydrated and amorphous cellulose.

For a $\mathrm{NaOH}$ concentration of $10 \%$ (Figure $2(\mathrm{a})$ ), the CI was still the most frequent allomorph. The crystallinity index decreased very slightly through the alkalization step. When the alkali concentration was 20\% (Figure 2(b)), the CI fraction decreased once the alkalization step started, whereas the percentage of CII increased from 0 to $90 \mathrm{~min}$. The conversion of CI into CII was faster for the highest $\mathrm{NaOH}$ 


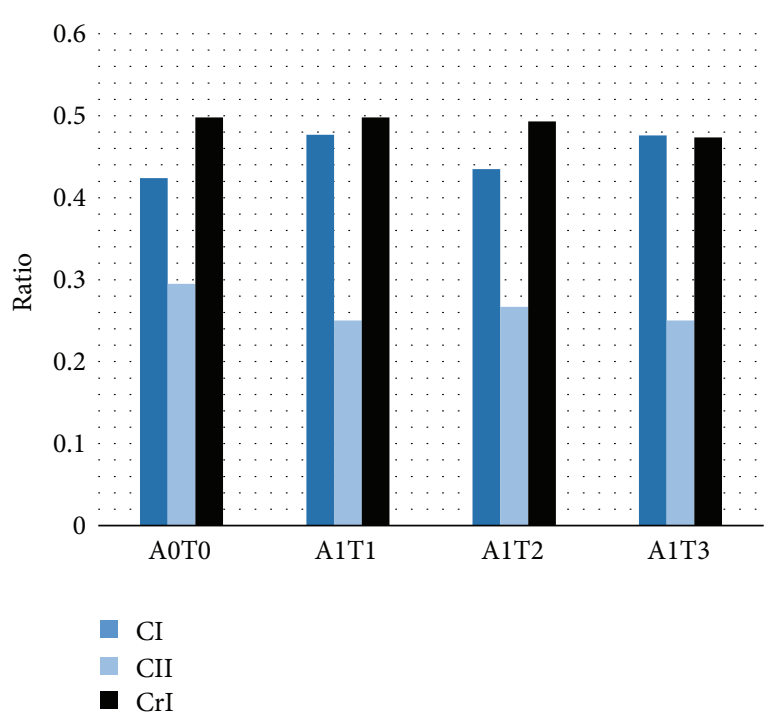

(a)

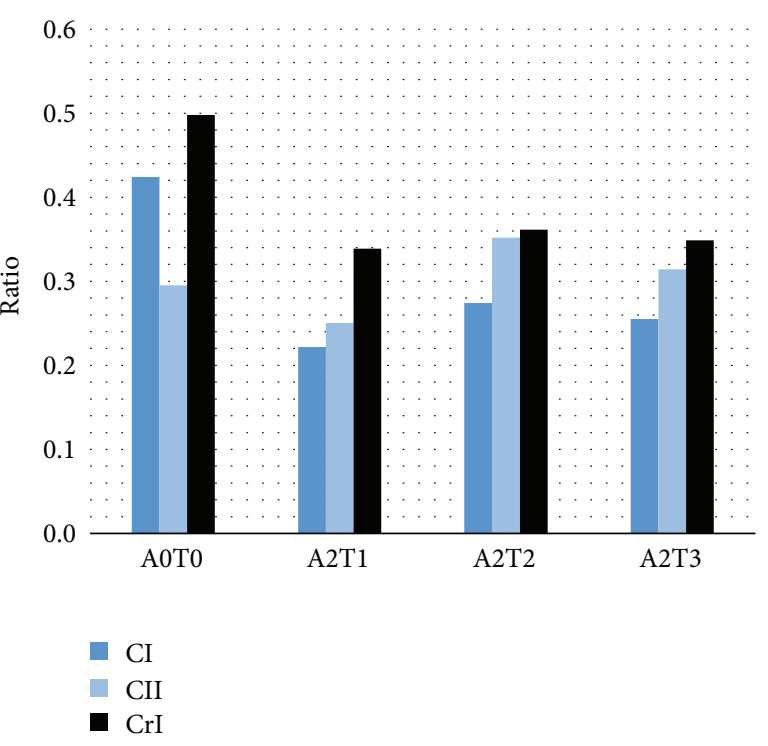

(b)

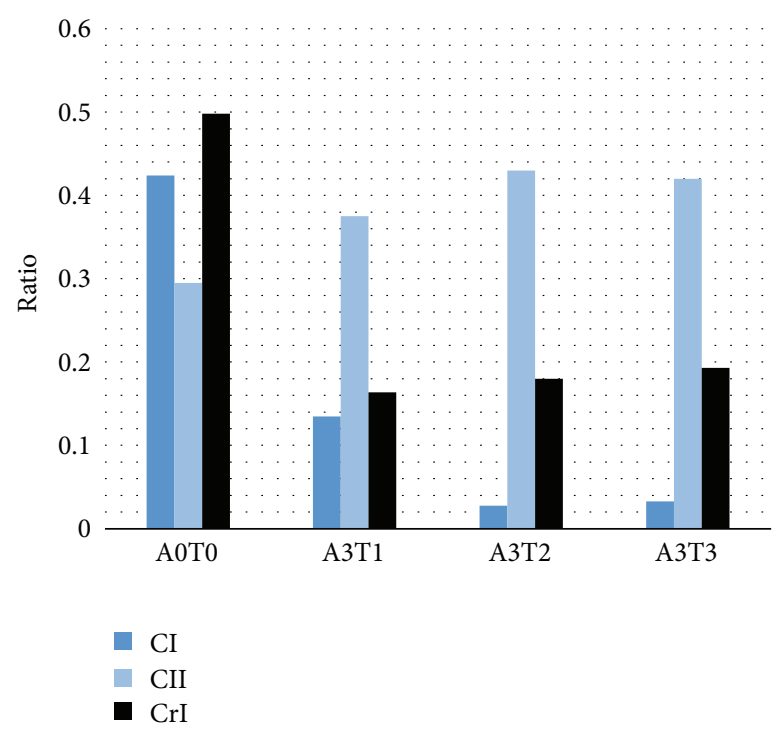

(c)

Figure 2: Crystallinity index and ratios of $\mathrm{CI}$ and $\mathrm{CII}$ for various $\mathrm{NaOH}$ concentrations and times. (a) $\mathrm{NaOH} 10 \%$. (b) $\mathrm{NaOH} 20 \%$. (c) $\mathrm{NaOH}$ $30 \%$.

concentration (Figure 2(c)). The crystallinity index decreased abruptly with $\mathrm{NaOH}$ concentration.

3.2. Elemental Analysis. The results were processed with various kinetic models and a pseudo-second-order rate equation was found to provide the best fit. The pseudosecond-order kinetic model proposed by Blanchard et al. [38] is typically applied to adsorption phenomena occurring in solution. These kinetic expressions have been applied to a variety of systems [39]. The theoretical background has been examined by Azizian [40]. The kinetic constants of pseudo-second-order models are combinations of the initial solute concentration and the adsorption and desorption parameters.
The proportion of EPTAC bound to cellulose in the cationization reaction was referred to that of elemental nitrogen fixed by cellulose. The driving force was the difference between the proportion of nitrogen fixed by cellulose at time $t$ and the maximum (saturation or equilibrium) proportion. The rate equation in terms of the proportion of elemental nitrogen fixed by cellulose was

$$
\frac{d N_{t}}{d t}=k\left(N_{\mathrm{eq}}-N_{t}\right)^{2}
$$

where $N_{t}$ is proportional to the number of active sites occupied by the active cationic group on cellulose at time $t$ and so is $N_{\text {eq }}$ to the number of sites available on cellulose 
fibres at equilibrium (both as percentages of elemental nitrogen).

Integration of this differential equation under the boundary conditions $t=0$ to $t=t$ and $N_{t}=0$ to $N_{t}=N_{t}$ yields

$$
N_{t}=\frac{N_{\mathrm{eq}}^{2} k t}{1+N_{\mathrm{eq}} k t},
$$

which is the integral form of the equation for a pseudosecond-order reaction. Expressing this equation in terms of the time/fixed nitrogen proportion ratio leads to a linear function of time.

The previous kinetic equation can be written in linear form as

$$
\frac{t}{N_{t}}=\frac{t}{N_{\mathrm{eq}}}+\frac{1}{k N_{\mathrm{eq}}} .
$$

The concentrations of nitrogen at different reaction times $t$ as determined by elemental analysis of dry cationized samples were used to plot the linearized form of the equation. If the assumption of a pseudo-second-order model is fulfilled, then the intercept of the curve will represent the highest proportion of elemental nitrogen incorporated by effect of the reaction of EPTAC, previously formed in the reaction between CHPTAC and $\mathrm{NaOH}$, with alkali-activated sites of the hydroxyl group on $\mathrm{C}_{6}$ in the anhydroglucose unit (AGU). Likewise, the slope of the curve will coincide with the reciprocal of the equilibrium (saturation) concentration. The figures of merit of the linearized equations as obtained by least-squares regression are shown in Figure 3. As can be seen, the coefficients of correlation ranged from 0.97 to 0.99 .

The dotted lines in Figure 4 represent the experimental percent nitrogen contents as measured by elemental analysis as a function of the reaction time for cellulose treated with $\mathrm{NaOH}$, whereas the solid lines represent the integrated kinetic equations in terms of parameters $N_{\text {eq }}$ and $K$ as calculated from the linearized equation: $t / N t$ versus $t$ (Table 2).

Table 2 also lists the root mean square deviation (RMSD) as a measure of differences between experimental values and predicted values (i.e., those obtained from the pseudosecond-order kinetic equation). The degree of nitrogen substitution (DNS) of the cellulose was calculated from the nitrogen content $(\% \mathrm{~N})$ and the molecular weight of the anhydroglucose unit (AGU), 162.15, using the following expression:

$$
\mathrm{NSD}=\frac{162.15 \times \% \mathrm{~N}}{1400-151.64 \times \% \mathrm{~N}}
$$

where $\% \mathrm{~N}$ denotes the percentage of dry elemental nitrogen, 1400 is 100 times the atomic weight of nitrogen, and 151.64 is the molecular weight of the epoxypropyltrimethylammonium chloride (EPTMAC) group added.

Comparing the results of this work with the results of de la Motte and Westman [41], under the conditions leading to maximal incorporation of nitrogen in cationic form, DNS was greater in this work: 0.404 (i.e., $40.4 \%$ ) at the maximum $\% \mathrm{~N}$ level.
We used a constant CHPTAC/AGU ratio, $\mathrm{NaOH}$ concentration, and isopropyl alcohol proportion throughout. DNS for cationic starches typically ranges from 0.0075 to 0.1215 and increases with increasing reaction time and proportion of cationizing reagent. Our results are consistent with those of other studies [42].

Based on the available literature, DNS increases with increase in the CHPTAC concentration; also, using an inadequate amount of $\mathrm{NaOH}$ hinders formation of the epoxide and alkoxides from the polysaccharide, whereas excess $\mathrm{NaOH}$ causes degradation of the epoxide and decreases the molecular weight of the product.

Figure 5 shows the variation of $N_{\mathrm{eq}}$ and $k$ with the amorphous fraction (AF) of cellulose (see (6)). $N_{\text {eq }}$ was linearly fitted to the amorphous faction (see (7)). Hence,

$$
\begin{gathered}
\mathrm{AF}=1-\mathrm{CI}-\mathrm{CII} \\
N_{\text {eq }}=6.94 \mathrm{AF}-0.996
\end{gathered}
$$

with $R^{2}=0.98$. Likewise, the best fit with the pseudo-secondorder rate constant $k$ was provided by

$$
k=12.32 \mathrm{AF}+4.84
$$

with $R^{2}=0.83$. Hence, the rate of nitrogen substitution was found to increase with increasing amorphous fraction. The more severe the conditions of the alkalization stage were, the faster the cationization reaction occurred and the higher the equilibrium value was.

\section{Conclusion}

Under the experimental conditions used in this work, the overall cationization reaction fits a pseudo-second-order kinetic equation. The reaction rate increases with the square of the difference between the equilibrium and the apparent concentrations of nitrogen. This squared difference decreases-and so does the reaction rate, in an asymptotic manner-as the amount of nitrogen fixed to cellulose approaches its equilibrium level.

As previously found for other chemical and enzymatic reactions, the reactivity of cellulose increases with decreasing crystallinity index and increasing amorphous fraction to a greater extent than it does with the proportion of cellulose II (CII), even though the amorphous fraction is related to CII.

The proportion of nitrogen at equilibrium in cationized cellulose increases linearly with increasing content of amorphous cellulose in the starting material. Initially, the reaction rate is comparatively high. As a result, the proportion of nitrogen reaches a near-equilibrium level within a short time (about $30 \mathrm{~min}$ ). To obtain cationic cellulose with a high substitution degree, it is advisable to have a previously performed alkalization stage under severe conditions. 
TABLE 2: Parameters of pseudo-second-order rate equations.

\begin{tabular}{lccccccccc}
\hline $\begin{array}{l}\text { Parameters of original } \\
\text { kinetic equation }\end{array}$ & A1T1 & A1T2 & A1T3 & A2T1 & A2T2 & A2T3 & A3T1 & A3T2 & A3T3 \\
\hline$N_{\text {eq }}, \% \mathrm{~N}$ & 0.11 & 0.15 & 0.17 & 0.13 & 0.15 & 0.19 & 0.67 & 1.56 & 1.79 \\
$\mathrm{DNS}_{\mathrm{eq}}$ & 0.013 & 0.018 & 0.020 & 0.015 & 0.018 & 0.022 & 0.084 & 0.217 & 0.257 \\
$N_{\mathrm{eq}}^{2} k, \% \mathrm{~N} / \mathrm{min}$ & 0.051 & 0.058 & 0.106 & 0.032 & 0.065 & 0.099 & 0.140 & 0.189 & 0.217 \\
$k,(\% \mathrm{~N} \cdot \mathrm{min})^{-1}$ & 4.13 & 2.69 & 3.54 & 1.93 & 2.99 & 2.87 & 0.32 & 0.08 & 0.07 \\
$\mathrm{RMSD} / 10^{-3}$ & 5.1 & 6.1 & 6.6 & 3.9 & 4.7 & 5.1 & 33 & 67 \\
\hline
\end{tabular}

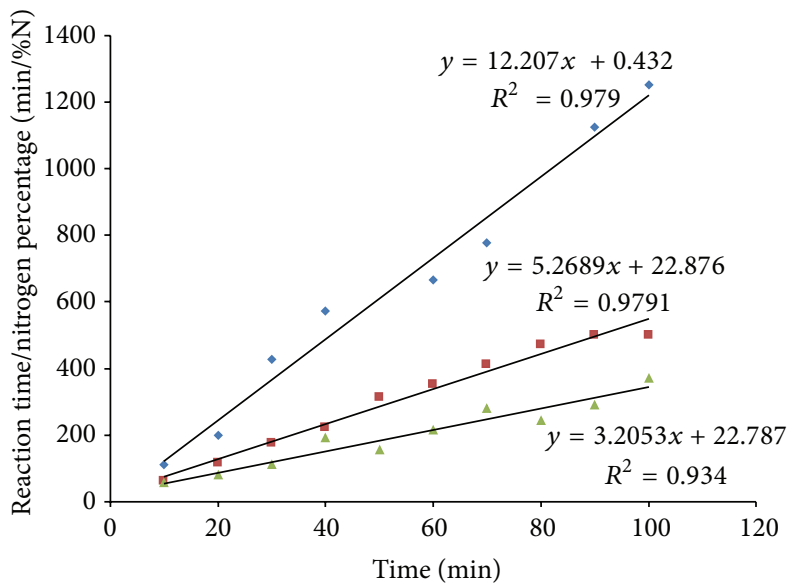

$30 \mathrm{~min}$

$60 \mathrm{~min}$

$\triangle 90 \mathrm{~min}$

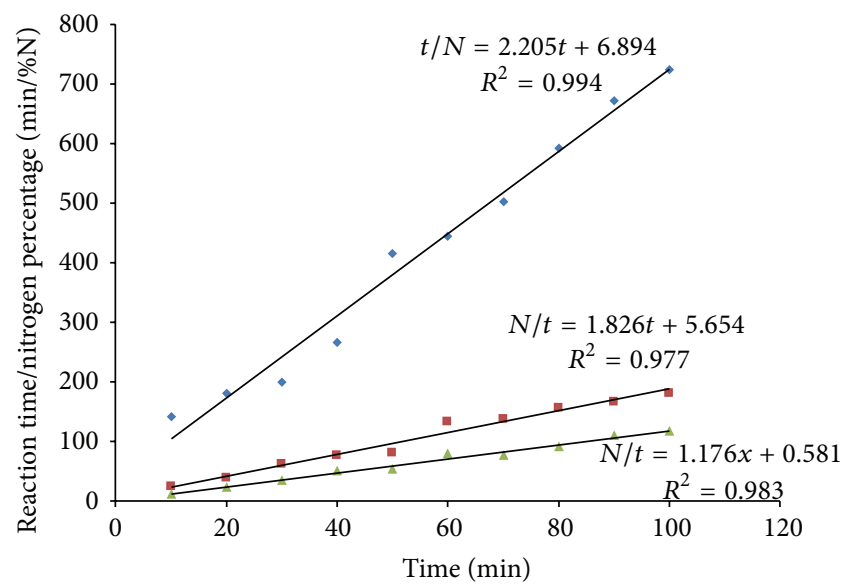

$30 \mathrm{~min}$

$60 \mathrm{~min}$

$90 \mathrm{~min}$

(a)

(b)

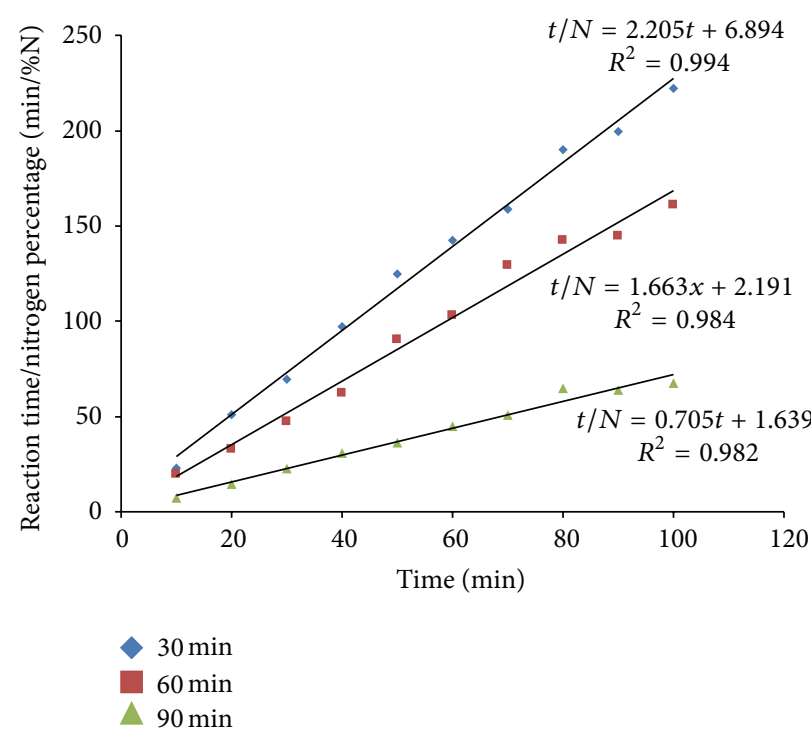

(c)

FIGURE 3: Linearized representation of the pseudo-second-order rate equation for cationized cellulose, previously alkalized with (a) NaOH $10 \%$, (b) $\mathrm{NaOH} 20 \%$, and (c) $\mathrm{NaOH} 30 \%$. 

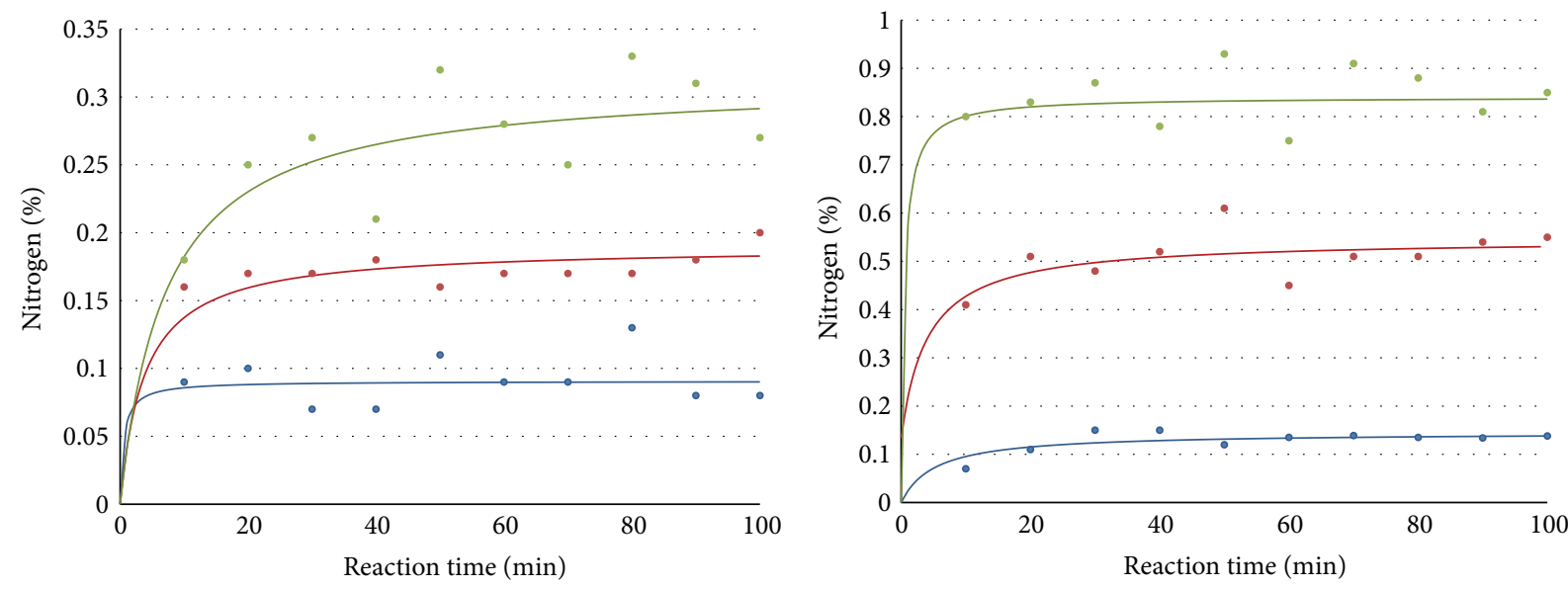

$$
\begin{aligned}
& \text { - } 90 \mathrm{~min} \\
& \text { - } 60 \mathrm{~min} \\
& \text { - } 30 \mathrm{~min}
\end{aligned}
$$

(a)

(b)

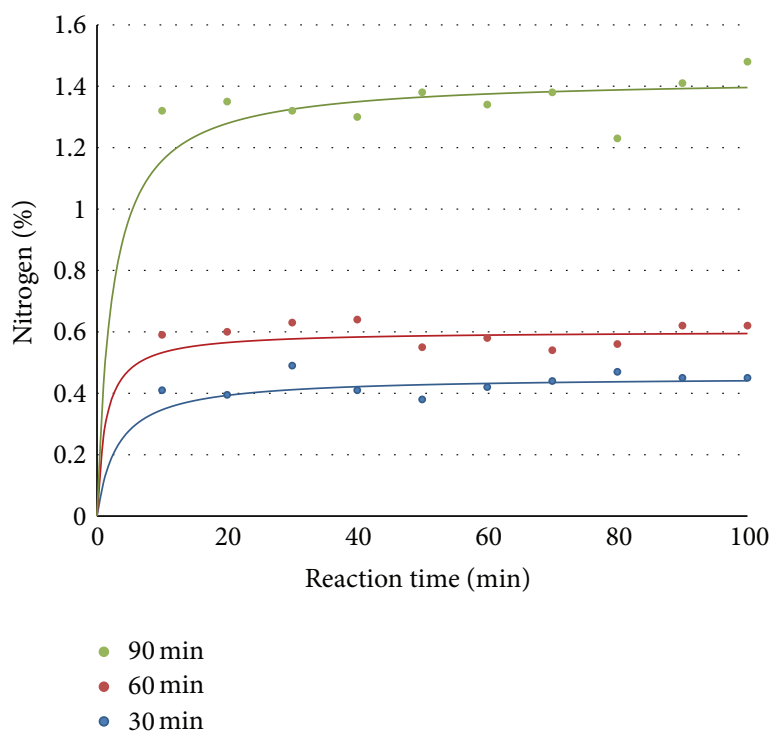

(c)

Figure 4: Percentage of nitrogen in cellulose after cationization following alkalization with aqueous solutions of (a) $\mathrm{NaOH} 10 \%$, (b) $\mathrm{NaOH}$ $20 \%$, and (c) $\mathrm{NaOH} 30 \%$.

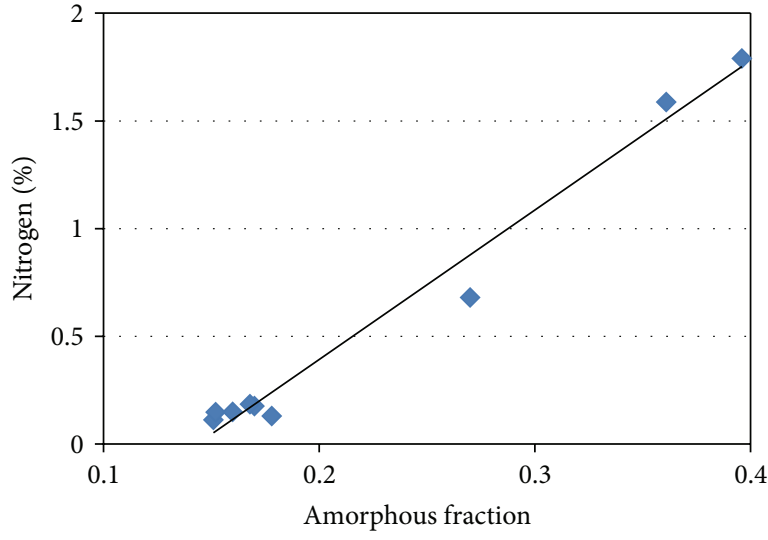

(a)

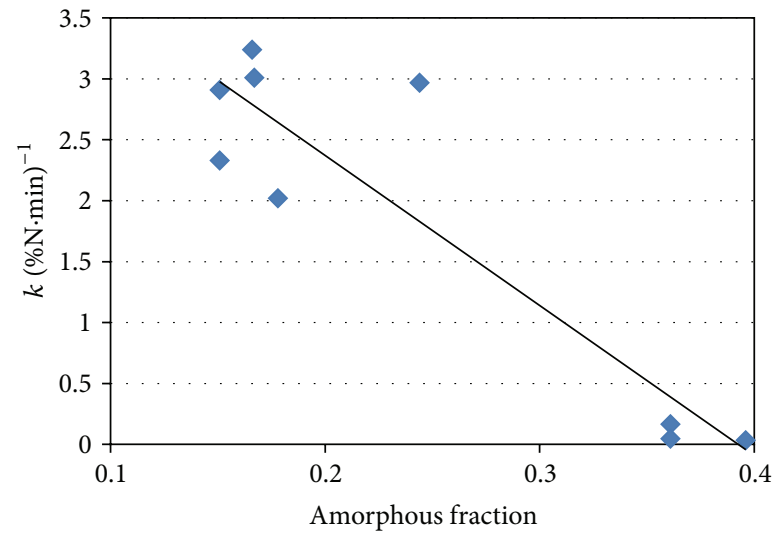

(b)

FIGURE 5: Nitrogen percentage (a) and kinetic constant of the pseudo-second-order model (b) versus amorphous fraction of cellulose. 


\section{Conflict of Interests}

The authors declare that there is no conflict of interests regarding the publication of this paper.

\section{Acknowledgment}

The authors wish to acknowledge the Ministry of Science and Innovation of Spain for the financial support of the Project CTQ2010-21660-C03-01, to which the present study is related.

\section{References}

[1] P. Mocchiutti and M. A. Zanuttini, "Key considerations in the determination of polyelectrolyte concentration by the colloidal titration method," BioResources, vol. 2, no. 3, pp. 399-407, 2007.

[2] M. A. Hubbe, A. Sundberg, P. Mocchiutti, Y. Ni, and R. Pelton, "Dissolved and colloidal substances (DCS) and the charge demand of papermaking process waters and suspensions: a review," BioResources, vol. 7, no. 4, pp. 6109-6193, 2012.

[3] Q. Miao, L. Huang, and L. Chen, "Advances in the control of dissolved and colloidal substances present in papermaking processes: a brief review," BioResources, vol. 8, no. 1, pp. 14311455, 2013.

[4] R. I. S. Gill, "Chemical control of deposits-scopes and limitations," Paper Technology, vol. 37, no. 6, pp. 23-31, 1996.

[5] J. Shen, Z. Song, X. Qian, and W. Liu, "Modification of papermaking grade fillers: a brief review," BioResources, vol. 4, no. 3, pp. 1190-1209, 2009.

[6] F. A. Adamsky and B. J. Williams, "Effects of new drainage, retention, end formation technology for improving production rates and runnability of recycled fiber cylinder machines," Tappi Journal, vol. 79, no. 8, pp. 175-182, 1996.

[7] H. Li, Y. Du, X. Wu, and H. Zhan, "Effect of molecular weight and degree of substitution of quaternary chitosan on its adsorption and flocculation properties for potential retentionaids in alkaline papermaking," Colloids and Surfaces A: Physicochemical and Engineering Aspects, vol. 242, no. 1-3, pp. 1-8, 2004.

[8] Z. Yan, Q. Liu, Y. Deng, and A. Ragauskas, "Improvement of paper strength with starch modified clay," Journal of Applied Polymer Science, vol. 97, no. 1, pp. 44-50, 2005.

[9] A. Swerin, G. Risinger, and L. Ödberg, "Flocculation in suspensions of microcrystalline cellulose by microparticle retention aid systems," Journal of Pulp and Paper Science, vol. 23, no. 8, pp. 374-381, 1997.

[10] S. Forsberg and G. Strom, "Effect of contact time between cationic polymers and furnish on retention and drainage," Journal of Pulp and Paper Science, vol. 20, no. 3, pp. J71-J76, 1994.

[11] H. J. Prado and M. C. Matulewicz, "Cationization of polysaccharides: a path to greener derivatives with many industrial applications," European Polymer Journal, vol. 52, no. 1, pp. 5375, 2014.

[12] M. Hashem, P. Hauser, and B. Smith, "Reaction efficiency for cellulose cationization using 3-chloro-2-hydroxypropyl trimethyl ammonium chloride," Textile Research Journal, vol. 73, no. 11, pp. 1017-1023, 2003.

[13] Y. Song, Y. Sun, X. Zhang, J. Zhou, and L. Zhang, "Homogeneous quaternization of cellulose in $\mathrm{NaOH} /$ Urea aqueous solutions as gene carriers," Biomacromolecules, vol. 9, no. 8, pp. 2259-2264, 2008.
[14] M. Hashem, M. El-Bisi, S. Sharaf, and R. Refale, "Precationization of cotton fabrics: an effective alternative tool for activation of hydrogen peroxide bleaching process," Carbohydrate Polymers, vol. 98, no. 2, pp. 1371-1376, 2013.

[15] M. Hashem, P. Hauser, and B. Smith, "Wrinkle recovery for cellulosic fabric by means of ionic crosslinking," Textile Research Journal, vol. 73, no. 9, pp. 762-766, 2003.

[16] Z. T. Liu, Y. Yang, L. Zhang, Z. W. Liu, and H. Xiong, "Study on the cationic modification and dyeing of ramie fiber," Cellulose, vol. 14, no. 4, pp. 337-345, 2007.

[17] M. Montazer, R. M. A. Malek, and A. Rahimi, "Salt free reactive dyeing of cationized cotton," Fibers and Polymers, vol. 8, no. 6, pp. 608-612, 2007.

[18] M. R. Kweon, P. R. Bhirud, and F. W. Sosulski, "An aqueous alcoholic-alkaline process for cationization of corn and pea starches," Starch, vol. 48, no. 6, pp. 214-220, 1996.

[19] M. S. Khalil-Abad, M. E. Yazdanshenas, and M. R. Nateghi, "Effect of cationization on adsorption of silver nanoparticles on cotton surfaces and its antibacterial activity," Cellulose, vol. 16, no. 6, pp. 1147-1157, 2009.

[20] M. Wu and S. Kuga, "Cationization of cellulose fabrics by polyallylamine binding," Journal of Applied Polymer Science, vol. 100, no. 2, pp. 1668-1672, 2006.

[21] H. J. Prado, M. C. Matulewicz, P. R. Bonelli, and A. L. Cukierman, "Studies on the cationization of agarose," Carbohydrate Research, vol. 346, no. 2, pp. 311-321, 2011.

[22] S. Pal, S. Ghosh, G. Sen, U. Jha, and R. P. Singh, "Cationic tamarind kernel polysaccharide (Cat TKP): a novel polymeric flocculant for the treatment of textile industry wastewater," International Journal of Biological Macromolecules, vol. 45, no. 5, pp. 518-523, 2009.

[23] V. K. Kaushik, A. Kumar, and S. Kalia, "Effect of mercerization and benzoyl peroxide treatment on morphology, thermal stability and crystallinity of sisal fibers," International Journal of Textile Science, vol. 1, no. 6, pp. 101-105, 2012.

[24] A. J. Stipanovic and A. Sarko, "Packing analysis of carbohydrates and polysaccharides. 6. Molecular and crystal structure of regenerated cellulose II," Macromolecules, vol. 9, no. 5, pp. 851857, 1976.

[25] C. Techapun, N. Poosaran, M. Watanabe, and K. Sasaki, "Thermostable and alkaline-tolerant microbial cellulase-free xylanases produced from agricultural wastes and the properties required for use in pulp bleaching bioprocesses: a review," Process Biochemistry, vol. 38, no. 9, pp. 1327-1340, 2003.

[26] H. Yamamoto and F. Horii, "CPMAS carbon-13 NMR analysis of the crystal transformation induced for Valonia cellulose by annealing at high temperatures," Macromolecules, vol. 26, no. 6, pp. 1313-1317, 1993.

[27] P. Zugenmaier, "Conformation and packing of various crystalline cellulose fibers," Progress in Polymer Science, vol. 26, no. 9, pp. 1341-1417, 2001.

[28] A. Jähn, M. W. Schröder, M. Füting, K. Schenzel, and W. Diepenbrock, "Characterization of alkali treated flax fibres by means of FT Raman spectroscopy and environmental scanning electron microscopy," Spectrochimica Acta Part A: Molecular and Biomolecular Spectroscopy, vol. 58, no. 10, pp. 2271-2279, 2002.

[29] E. Dinand, M. Vignon, H. Chanzy, and L. Heux, "Mercerization of primary wall cellulose and its implication for the conversion of cellulose I $\rightarrow$ cellulose II," Cellulose, vol. 9, no. 1, pp. 7-18, 2002 . 
[30] F. J. Kolpak and J. Blackwell, "Determination of the structure of cellulose II," Macromolecules, vol. 9, no. 2, pp. 273-278, 1976.

[31] Y. Nishiyama, P. Langan, and H. Chanzy, "Crystal structure and hydrogen-bonding system in cellulose I $\beta$ from synchrotron $\mathrm{X}$-ray and neutron fiber diffraction," Journal of the American Chemical Society, vol. 124, no. 31, pp. 9074-9082, 2002.

[32] Y. Nishiyama, J. Sugiyama, H. Chanzy, and P. J. Langan, “Crystal structure and hydrogen bonding system in cellulose $\mathrm{I}_{\alpha}$ from synchrotron X-ray and neutron fiber diffraction," Journal of the American Chemical Society, vol. 125, no. 47, pp. 14300-14306, 2003.

[33] H. A. Krässig, "Methods of fiber structure characterization," in Cellulose: Structure, Accessibility and Reactivity, vol. 11 of Polymer Monographs, pp. 88-125, Gordon and Breach Science, Yverdon, Switzerland, 1993.

[34] A. Moral, R. Aguado, and A. Tijero, "Cationization of native and alkalized cellulose: mechanism and kinetics," Cellulose Chemistry and Technology. In press.

[35] E. Gümüskaya, M. Usta, and H. Kirci, "The effects of various pulping conditions on crystalline structure of cellulose in cotton linters," Polymer Degradation and Stability, vol. 81, no. 3, pp. 559-564, 2003.

[36] S. Park, J. O. Baker, M. E. Himmel, P. A. Parilla, and D. K. Johnson, "Cellulose crystallinity index: measurement techniques and their impact on interpreting cellulase performance," Biotechnology for Biofuels, vol. 3, article 10, 2010.

[37] S. Y. Oh, D. Yoo, Y. Shin et al., "Crystalline structure analysis of cellulose treated with sodium hydroxide and carbon dioxide by means of X-ray diffraction and FTIR spectroscopy," Carbohydrate Research, vol. 340, no. 15, pp. 2376-2391, 2005.

[38] G. Blanchard, M. Maunaye, and G. Martin, "Removal of heavy metals from waters by means of natural zeolites," Water Research, vol. 18, no. 12, pp. 1501-1507, 1984.

[39] W. Rudzinski and W. Plazinski, "On the applicability of the pseudo-second order equation to represent the kinetics of adsorption at solid/solution interfaces: a theoretical analysis based on the statistical rate theory," Adsorption, vol. 15, no. 2, pp. 181-192, 2009.

[40] S. Azizian, "Kinetic models of sorption: a theoretical analysis," Journal of Colloid and Interface Science, vol. 276, no. 1, pp. 47-52, 2004.

[41] H. de la Motte and G. Westman, "Regioselective cationization of cellulosic materials using an efficient solvent-minimizing spraytechnique," Cellulose, vol. 19, no. 5, pp. 1677-1688, 2012.

[42] S. Radosta, W. Vorwerg, A. Ebert, A. H. Begli, D. Grülc, and M. Wastyn, "Properties of low-substituted cationic starch derivatives prepared by different derivatisation processes," Starch, vol. 56, no. 7, pp. 277-287, 2004. 

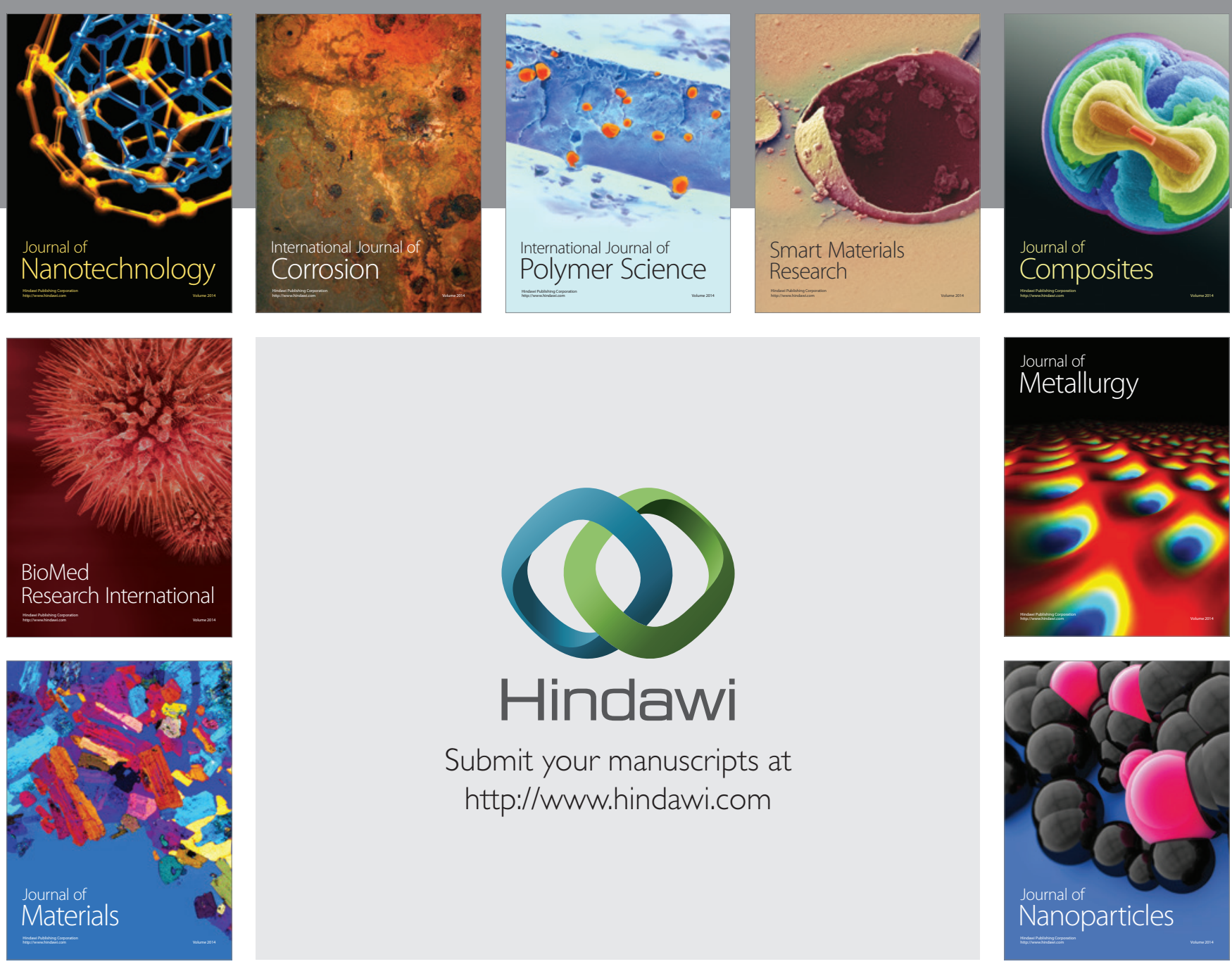

Submit your manuscripts at http://www.hindawi.com
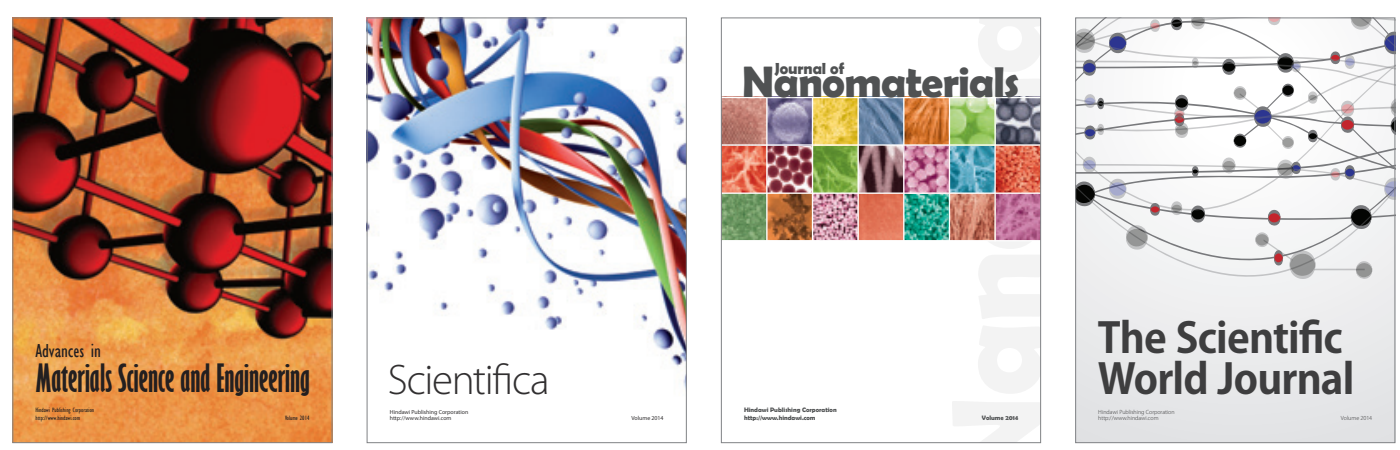

\section{The Scientific World Journal}
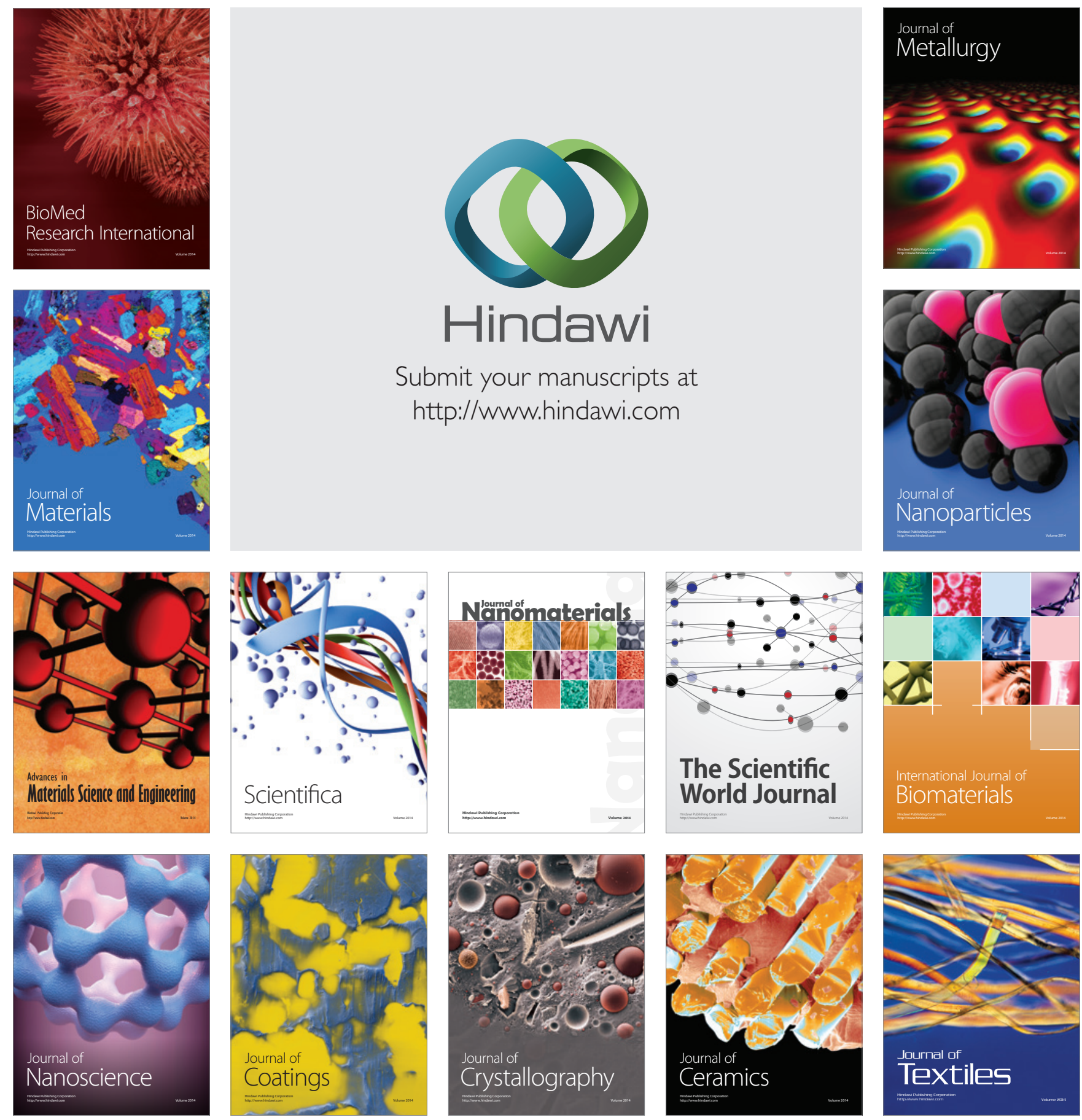